\title{
14. Kurs für Kardiovaskuläre Nuklearmedizin/Nuklearkardiologie
}

Vom 12. bis zum 14. September 2019 fand in Potsdam der 14. Kurs für Kardiovaskuläre Nuklearmedizin/Nuklearkardiologie unter Schirmherrschaft der Deutschen Gesellschaft für Nuklearmedizin und der Deutschen Gesellschaft für Kardiologie statt. Am sehr geeigneten Kongressort im Inselhotel Potsdam ( $\triangleright$ Abb. 1) trafen sich 135 Teilnehmer, darunter 112 Fachteilnehmer und 23 Repräsentanten der Industrie. 21 Referenten ( 7 aus dem Bereich der Kardiologie, 2 aus der Radiologie sowie 12 Nuklearmediziner) präsentierten 22 Beiträge, davon 12 mit TED-Fragen, und 3 Sitzungen mit interessanten Falldemonstrationen.

Beeindruckend waren die hohe Qualität und Aktualität der Beiträge sowie die angeregte Diskussion derselben im Plenum. Die Interdisziplinarität der Referenten und auch der Teilnehmer, von denen ein erheblicher Anteil jüngeren Alters waren, schufen eine durch und durch positive Arbeitsatmosphäre.

Am Freitag wurde der aktuelle Stand der Nuklearkardiologie in Hinblick auf die Häufigkeit und Art der Durchführung der Myokard-Perfusions-Szintigrafie (MPS), die Evidenz aus Multizentrischen Studien, ein Überblick über die aktuellen nationalen und internationalen Leitlinien sowie die

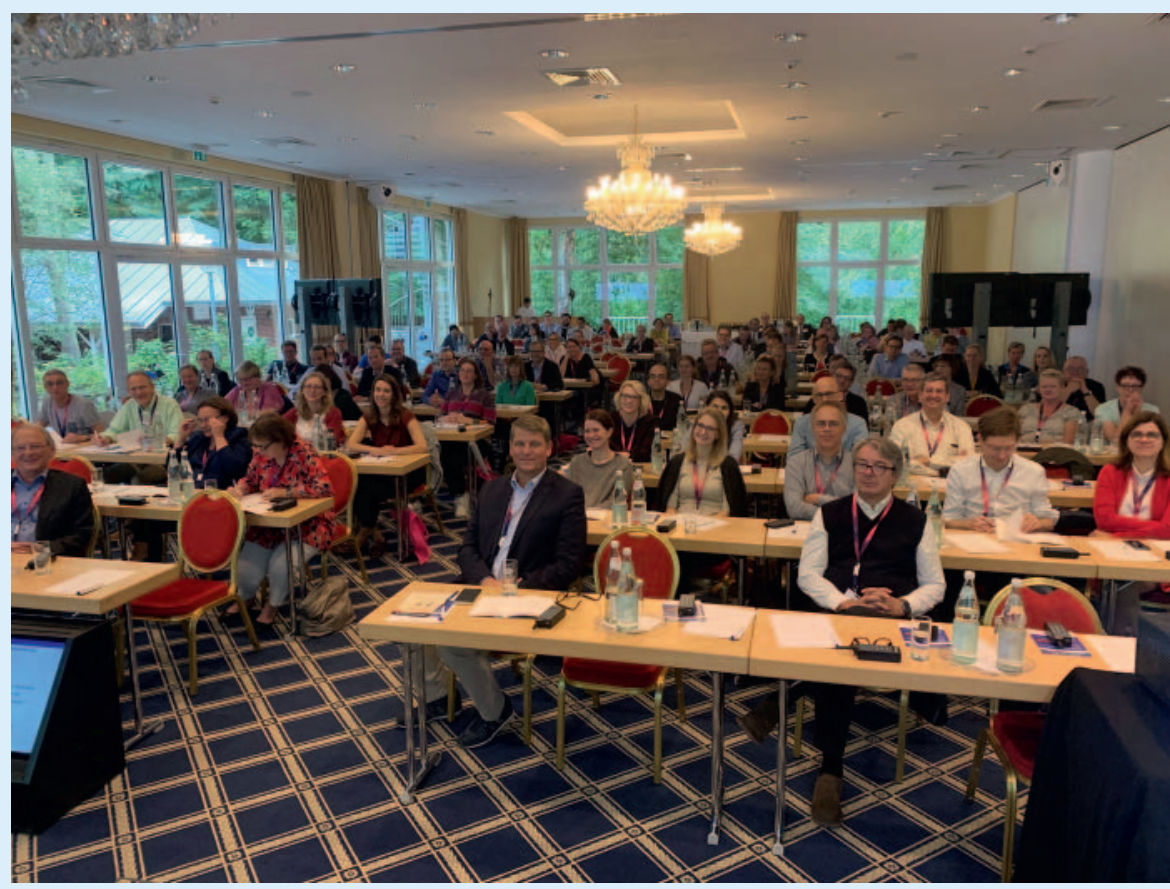

- Abb. 1 14. Kurs für Kardiovaskuläre Nuklearmedizin/Nuklearkardiologie im Inselhotel Potsdam (Foto: Prof. Dr. Wolfgang Burchert, Bad Oeynhausen).

aktuellen Belastungsverfahren referiert. Es folgte dann eine Sitzung mit neuen technischen Entwicklungen im klinischen Einsatz wie Präsentationen zu CZT-Kameras D-SPECT und Discovery 530NM sowie das IQ-Paket für SPECT und SPECT/CT-Systeme.
Allen gemeinsam ist eine bessere Zählratenausbeute und damit die Möglichkeit, die Untersuchungszeit deutlich zu verkürzen und/oder die Strahlendosis deutlich einzusparen. 
Ein besonderer Schwerpunkt der Veranstaltung war die Diagnostik des Herzens bei Infektionen/Inflammation und Speicherkrankheiten. Bei entzündlichen Erkrankungen wurde zunächst ein Überblick zur metabolischen Vorbereitung der FDG-PET im Kontext der Entzündungsbildgebung präsentiert. Bei Endokarditis und DeviceInfekt wurde der Einsatz von SPECT und PET demonstriert. Die Diagnostik der Herzbeteiligung bei Sarkoidose mit der FDGPET/CT - auch im Vergleich mit der MRT war ein weiterer Schwerpunkt der Tagung. Ein neueres Thema war der Nachweis der kardialen Beteiligung bei einer Amyloidose. Mit der bewährten Skelettszintigrafie gelingt dies bei der ATTR-Form, für die anderen Subformen sind die PET-Amyloidose-Tracer in vielen Fällen geeignet.

Am Samstag wurde in Potsdam der aktuelle Stand unterschiedlicher Verfahren zur Ischämie-Diagnostik dargestellt. Das Belastungs-
EKG ist hier nur noch in Ausnahmesituationen geeignet. Gut einsetzbar im Alltag ist die Myokardszintigrafie (10\%-Schwelle als eine Indikation für die Revaskularisation), welche auch in der gesetzlichen Krankenversorgung abgebildet ist. In der Stressechokardiografie sind Abrechnungsprobleme und auch die Patienten-/Untersucherabhängigkeit eine Begrenzung für den breiten Einsatz im Alltag. Die unzureichende Möglichkeit der Abrechenbarkeit in der GKV verhindert den intensiven klinischen Einsatz der MRT und der CT-Koronarangiografie bei ansonsten guter klinischer Performance. Die neuen ESC-Leilinien für das chronische Koronarsyndrom (CCS) betonen bei mittlerer Prä-TestWahrscheinlichkeit die Bedeutsamkeit der nichtinvasiven Bildgebung, wobei die Koronar-CT bei niedriger Prä-Test-Wahrscheinlichkeit zum Krankheitsausschluss eine besondere Bedeutung bekommen hat.
Unter dem Titel „Was gibt's Neues?“ wurden zudem Bereiche wie kardiale Medikamente, Lungendiagnostik, Herzinsuffizienzdiagnostik, Indikationen zur PET-MRT und Aktuelles aus der Berufspolitik vorgetragen.

Allerherzlichster Dank gilt den Mitarbeitern von der vokativ $\mathrm{GmbH}$ für die hervorragende Organisation der Veranstaltung. Vielen Dank auch der Industrie für die materielle Unterstützung und die Informationen über Pharmaka und Medizingeräte. Den Hauptorganisatoren ist besonderer Dank geschuldet für die Initiierung, die Gestaltung des Programms sowie deren Ausführung. Dieser Dank richtet sich an die Kollegen Professor Schäfer, Dr. Dörr, Professor vom Dahl, PD Rischpler, Professor Zimmermann und die OÄ Frau Dr. Knollmann. Eine rundum gelungene Veranstaltung auf hohem wissenschaftlichem Niveau!

Prof. Dr. Wolfgang Burchert, Bad Oeynhausen 